\title{
Auszug aus einem Schreiben des Herrn Doctors und Ritters Olbers an den Herausgeber. Bremen 1829. Sept. 28.
}

Am 26sten dieses kurz ror Mitternacht ist hier eine Feuerkugel von mälsiger Gröfse gesehen worden. Personen, die sich grade im Freyen befanden, sahen plötzlich alles wie durch einen äufserst hellen Blitz mit Tageshelle erleuchtet, und wurden aufljlickend einer Feuerkugel gewahr, die sich fast perpendiculär auf den Horizont zu stïzzen schien, aber noch über \& Aquilae nicht zersprang, sondern verlöschte. Ich selbst habe den Anblick dieser Feuerkugel um wenige Secunden verfehlt: aber den über 6 Minuten Etehenbleibenden sehr hellen milchfarbenen Schweif um so genauer gesehen. Grade beschäftiget, bey dem sehr heitern Himmel meine Zeit durch Sternverschwindungen zu berichligen, hatte ich um 11h $39^{\prime} 47^{\prime \prime}$ mittl. Zeit das Verschwinden von 71 Herculis beobachtet und trat nun cinige Mimuten vor dem Verschwinden von 78 IIerculis wieder auf mein Observationszimmer (etwa um $11^{\text {h }} 44^{\prime} \mathrm{MI}$. Z.), als ich zu meiner
Verwunderung a Aquilae als einen schünen Cometen mit einem schmalen, aber selır hellen grade in die Höhe gerichteten Schweif erblickte. Dieser stehend gebliebene Schweif der Feuerkugel war anfangs ganz grade, lrümmle und schlängelte sich indes nachler mohr und mehr, und schicn sich, doch sehr langsam, ein wenig aufwärls zu ziehen. Die Axe dieses Schweifs lag auf einen gröfsten Kreis, den man sich von $\propto$ Aquil. milten zwischen $\rho$ Aquil. und y Sag. durchgezogen vorstellen kann. Sein unteres Ende, dals sich dünn verlief, hörle noch ein paar Grade ïber $\alpha$ Aquil. etwa bey $\varphi$ Aquil. um $11^{\mathrm{h}} 48^{\prime} \mathrm{M}$. Z. auf, aber scin oberer Theil, mehr plötzlich abgeschnitten, reichte noch ïber z Sagiltae hinaus. Ich wïnsche sehr, dafs man dies Meteor auch an. derweitig gesehen und beobachitet haben möge, damit man den Abstand desselben von der Erd-Oberfäche genau bcrechnen liönue.

\section{W. Olbers.}

\section{Schreiben des Herrn Professors David an den Herausgeber. Prag 1829. Octbr. 21.}

Qo ungemein schlecht und ungünstig die Witterung vor dem 15 Octbr. war, so heiterte sich doch der Himmel beim Eintritte des $\alpha$ Tauri anf, und wir haben den Eintritt gut und genau beobachtet. Gegen 8 Uhr auch den Austritt des $2 \theta$ Tauri aus dem dunkeln Mondrande bey ciner Oeffnung der Wolken.

Den Austrilt \& Taiui haben wir wegen Wolken zu spät gesehen. Prof. Hallaschka beobachtele zu Beżeźan, 2 Meilen südöstlich von Prag, sowohl den Eintritt als Austritt, und wird Sie um horrespondirende Beobachtungen ersuchen. Das iiberstrümende Mondlicht bedeckte das $\propto$ Tauri so, dafs es 2-3 Zeitsekunden am lichten Rande zu hangen schien. Als die Hälfte des schwachen Sternlichtes zur Hälfte am lichten Rande answärts, zur Hälfte cinwä̀ls erschien, verschwand der Stern mit Schlag der Uhrsekunde mir, dem Prof. Bitıner und Diencr der Sternwarte Anton WFatzel. Bey der Beobachtung findet keine Unrichligkeit $\frac{x}{4} "$ stall; allein die Zeilbestimmung könnte wegen schlechter Wilterung zur Zeitbestimmung um $\frac{x}{3} "$ unrichlig seyn. Bey $\Lambda n$ gabe der millem Zeit liegt die Zeilgleichung aus Ihren Hülfstafeln zum Grunde.

1829 den 15 October. Austritt des $2 \theta$ Tauri aus dem dunkeln Mondrande nach mittlerer Zeit um $7^{\text {h }} 43^{\prime} 19 \frac{4}{x^{\circ}}$ ". Eintritt des $\alpha$ Tauri in den lichten Mondrand um 10 424 $\frac{3}{\mathrm{r}}$.

Beym Austritt bedeckten vorüberziehende Wolken den Mond.

Prof. David.

\section{I $\quad$ n}

Beobachtungen des Ganges einer Kesselsschen Pendeluhr und Bemerkungen darüber, von Herrn Prof. und Ritter Bessel. p. 1. Auszng aus einem Schreiben des Herm Doctors und Ritters Olbers an den Herausgeber. p. 15. Schreiben des Herrn Professor David an den Herausgeber. p. 15. 